\title{
[18F] FDOPA PET may confirm the clinical diagnosis of Parkinson's disease by imaging the nigro-striatal pathway and the sympathetic cardiac innervation: Proof-of-concept study
}

Jonathan Kuten ${ }^{1, *}$, Adi Linevitz ${ }^{2}$, Hedva Lerman ${ }^{1,2}$, Nanette Freedman ${ }^{1}$, Meir Kestenbaum ${ }^{2,3, \uparrow}$, Tamara Shiner $^{2,3,4}$ Nir Giladi ${ }^{2,3,4}$ and Einat Even-Sapir ${ }^{1,2}$

\author{
${ }^{1}$ Department of Nuclear Medicine, Tel-Aviv Sourasky Medical Center, Tel-Aviv, 6423906, Israel \\ ${ }^{2}$ Sackler Faculty of Medicine, Tel-Aviv University, Tel-Aviv, 6997801, Israel \\ ${ }^{3}$ Movement Disorders Unit, Neurological Institute, Tel-Aviv Sourasky Medical Center, Tel-Aviv, 6423906, Israel \\ ${ }^{4}$ Sagol School of Neurosciences, Tel-Aviv University, Tel-Aviv, 6997801, Israel \\ *Correspondence: jonathanku@tlvmc.gov.il (Jonathan Kuten) \\ "Current Address: Department of Neurology, Meir Medical Center, Kfar Saba, 4428164, Israel
}

\section{DOI: $10.31083 /$ i.jin.2020.03.196}

This is an open access article under the CC BY 4.0 license (https://creativecommons.org/licenses/by/4.0/).

Autonomic involvement, including cardiac denervation, may precede the motor symptoms of Parkinson's disease by several years. L-3,4-dihydroxy-6-[18F] fluorophenylalanine is a positron emitter and a true analog of L-dopa, used in clinical practice to assess striatal dopaminergic integrity. The present study aimed to assess the feasibility of evaluating cardiac sympathetic denervation in Parkinson's disease patients using L-3,4-dihydroxy6-[18F] fluoro-phenylalanine positron emission tomography/computed tomography. Patients referred for an L-3,4dihydroxy-6-[18F] fluoro-phenylalanine positron emission tomography/computed-tomography between July 2015 and May 2017 to evaluate striatal presynaptic dopaminergic integrity underwent a heart positron emission tomography scan following a brain positron emission tomography scan. L-3,4-dihydroxy-6-[18F] fluoro-phenylalanine uptake in the left ventricle was quantified using Carimas ${ }^{T M}$ software and compared between patients with and without Parkinson's disease. The area under the receiver operating characteristic curve was used to evaluate the ability of the left ventricular mean standardized uptake value to discriminate between patients with Parkinson's disease and those with other extrapyramidal syndromes. Seventysix patients were included, of whom 52 were diagnosed with Parkinson's disease. The mean L-3,4-dihydroxy-6[18F] fluoro-phenylalanine left ventricular mean standardized uptake value was lower in the Parkinson's disease patients compared to the non- Parkinson's disease patients $(1.08 \pm 0.21$ vs. $1.24 \pm 0.32, P=0.015)$. The left ventricular mean standardized uptake value was able to discriminate between Parkinson's disease and non- Parkinson's disease patients (the area under the receiver operating characteristic curve $=0.641, P=0.049$ ). In con- clusion, quantification of cardiac L-3,4-dihydroxy-6-[18F] fluoro-phenylalanine uptake may be able to differentiate between patients with and without Parkinson's disease. Validation of this finding in more substantial, prospective trials are warranted.

\section{Keywords}

Parkinson's disease; positron emission tomography; myocardium; neuroimaging

\section{Introduction}

Non-motor abnormalities, including those related to autonomic failure, may precede motor signs and symptoms of Parkinson's disease (PD) by years (Goldstein et al., 2002; Koller, 1992). Among the non-motor manifestations of PD, dementia, loss of sense of smell (anosmia), REM sleep behavior disorder, and orthostatic hypotension $(\mathrm{OH})$ have all been associated with cardiac sympathetic noradrenergic denervation (Goldstein et al., 2010; Kim et al., 2017; Miyamoto et al., 2006). Cardiac sympathetic denervation in PD as well as in other forms of synucleinopathies (i.e., dementia with Lewy bodies and pure autonomic failure) is explained by neurodegeneration of catecholaminergic post-ganglionic neurons resulting from deficient vesicular storage of catecholamines and accumulation of toxic byproducts, known as the "catecholaldehyde hypothesis" (Goldstein et al., 2011, 2014).

Cardiac sympathetic denervation has been histologically confirmed by decreased tyrosine hydroxylase immunoreactivity in epicardial nerves in patients with PD and Lewy body disease (Orimo et al., 2005; Orimo, 2002). The staging concept introduced by Braak et al. (2004) suggests that degeneration of the autonomic nervous system in PD precedes dopaminergic cell degeneration at the level of the substantia nigra, and thus cardiac sympathetic denervation may be a prodromal biomarker of PD (Goldstein 
et al., 2007; Oka et al., 2006, 2011; Orimo et al., 2005). Others have shown that cardiac sympathetic denervation in PD occurs independently of the movement disorder and progresses over time. Thus it may precede the motor symptoms by years or appear as a later finding (Goldstein et al., 2011; Orimo et al., 2008). Cardiac sympathetic denervation in PD can be visualized by means of sympathetic neuroimaging with investigational positron emission tomography (PET) agents, such as 6-[18F] fluorodopamine (Goldstein and Holmes, 1997) and [11C]-hydroxyephedrine (Raffel et al., 2006), as well as with the routinely clinically used singlephoton emission computed tomography (SPECT) agent [123I]metaiodobenzylguanidine ([123I]-MIBG) (Iwasa et al., 1998; Sudmeyer et al., 2011; Uyama et al., 2017; Yoshii et al., 2017).

Most patients with multiple system atrophy (MSA) have normal findings on neuroimaging studies of cardiac sympathetic innervation due to a preganglionic lesion rather than postganglionic involvement (Braune, 2001; Druschky et al., 2000; Orimo et al., 2001). As such, cardiac sympathetic neuroimaging can help differentiate between PD and MSA. This is especially useful in the case of PD with $\mathrm{OH}$, which almost invariably demonstrates sympathetic cardiac denervation (Goldstein et al., 2008, 2015). It has also been shown that cardiac sympathetic denervation may predict the development of PD in individuals with multiple risk factors for PD years before motor symptoms have developed (Goldstein et al., 2018). Other applications of imaging cardiac sympathetic denervation include assisting in differentiation between dementia with Lewy bodies from Alzheimer's disease in patients without Parkinsonism, imaging diabetic autonomic neuropathy, as well as evaluating the risk of sudden cardiac death in patients with heart failure (Jacobson et al., 2010; Manabe et al., 2017).

L-3,4-dihydroxy-6-[18F] fluoro-phenylalanine ([18F] FDOPA) is a positron emitter and a true analog of Ldihydroxyphenylalanine (L-dopa), the immediate precursor of dopamine and norepinephrine (Nanni et al., 2007). L-dopa is carried into the brain by the large neutral amino acid transport system, converted into dopamine by aromatic L-amino acid decarboxylase, and then stored in intraneuronal vesicles, from which it is released when the nerve cell fires (Garnett et al., 1983; Lovenberg et al., 1962).

[18F] FDOPA PET is an established imaging modality for the assessment of striatal dopaminergic integrity in neurodegenerative disorders, such as PD. The present study aimed to assess whether [18F] FDOPA can be used to evaluate cardiac sympathetic denervation for differentiating between patients with and those without PD by quantifying cardiac [18F] FDOPA uptake.

\section{Methods}

\subsection{Patients}

This study was approved by the local institutional ethics committee of a large tertiary university-affiliated medical center (approval number 0606-14-TLV) and registered as a clinical trial (ID code: NCT02495649). Neurologists referred the study patients for the evaluation of striatal presynaptic dopaminergic integrity because the clinical diagnosis was not conclusive. The participants underwent an [18F] FDOPA PET/CT scan of the brain in the medical center's Department of Nuclear Medicine between July 2015 and May 2017. They all signed a written consent before participating in the trial. The patients completed questionnaires about any past and current heart conditions, diabetes mellitus, and current medications. Antiparkinsonian medications were withheld for 24 hours before undergoing PET/CT. Fasting was not required, and carbidopa premedication was not used.

\subsection{Imaging protocol}

Patients were injected with 444-555 MBq (12-15 mCi) of [18F] FDOPA.

After a mean uptake time of $90.4 \pm 24.2$ minutes, the brain was scanned employing the Discovery 690 PET/CT system (GE Healthcare) with the head placed in a dedicated holder (Dhawan et al., 2002; Eshuis et al., 2006), followed immediately by acquiring a PET/CT scan of the heart with the same CT and PET acquisition parameters. The CT scan was acquired in a helical mode with automatic mA-modulation and $120 \mathrm{kV}$ voltage, and each CTscan was reconstructed to a slice thickness of $2.5 \mathrm{~mm}$. The static three-dimensional (3D) PET acquisition time was 7 minutes. PET images were acquired to obtain a $256 \times 256$ matrix with a pixel size of $1.2 \mathrm{~mm}$ and a total of 47 slices.

The reconstruction method was VUE Point FX by GE Healthcare that uses the time of flight information and includes a fully 3D OSEM algorithm. Six iterations, 32 subsets, and a filter cutoff of $5 \mathrm{~mm}$ were used for brain PET studies, and four iterations, 24 subsets, and a filter cutoff of $6.4 \mathrm{~mm}$ were used for cardiac PET studies. The VUE Point FX algorithm also includes normalization and image corrections for attenuation, scatter, randoms, and wasted time. A standard Z-filter was applied to smooth between transaxial slices.

None of the patients failed to tolerate the scan, and no adverse reactions were encountered.

\subsection{Image analysis}

The brain scans were read on a Xeleris workstation (GE Healthcare). They were qualitatively interpreted based on a visual assessment of [18F] FDOPA uptake in the basal ganglia by three nuclear medicine physicians (JK, HL, and EES) separately and then in consensus. The readers were blinded to the cardiac scans. Brain scans were considered positive for PD when there was decreased [18F] FDOPA activity in the basal ganglia in a pattern typical for PD (i.e., asymmetric decreased activity in a rostrocaudal pattern with relative sparing of the caudate nucleus). [18F] FDOPA uptake in the left ventricular myocardium was quantitatively analyzed using Carimas ${ }^{T M}$ software (Turku PET Centre, Finland) (Nesterov et al., 2009). This was carried out by a nuclear medicine physician (JK) and a nuclear medicine physicist (NF). The mean standardized uptake values (LV-SUV mean $_{\text {mere }}$ ) wen extracted. Fig. 1 shows an example of left ventricle myocardium segmentation using Carimas ${ }^{T M}$ software.

\subsection{Clinical diagnosis}

A clinical diagnosis of PD or non-PD was made by neurologists specializing in movement disorders (TS, MK), based on the UK brain bank criteria (Hughes et al., 1992) and after review of the patients' medical records for symptoms of idiopathic PD including motor parkinsonism as well as RBD, hyposmia, and brain $[18 \mathrm{~F}]$ FDOPA PET/CT findings. Non-PD patients were diagnosed as healthy individuals, or as having essential tremor (ET) or druginduced Parkinsonism. None had MSA or dementia with Lewy bodies. 

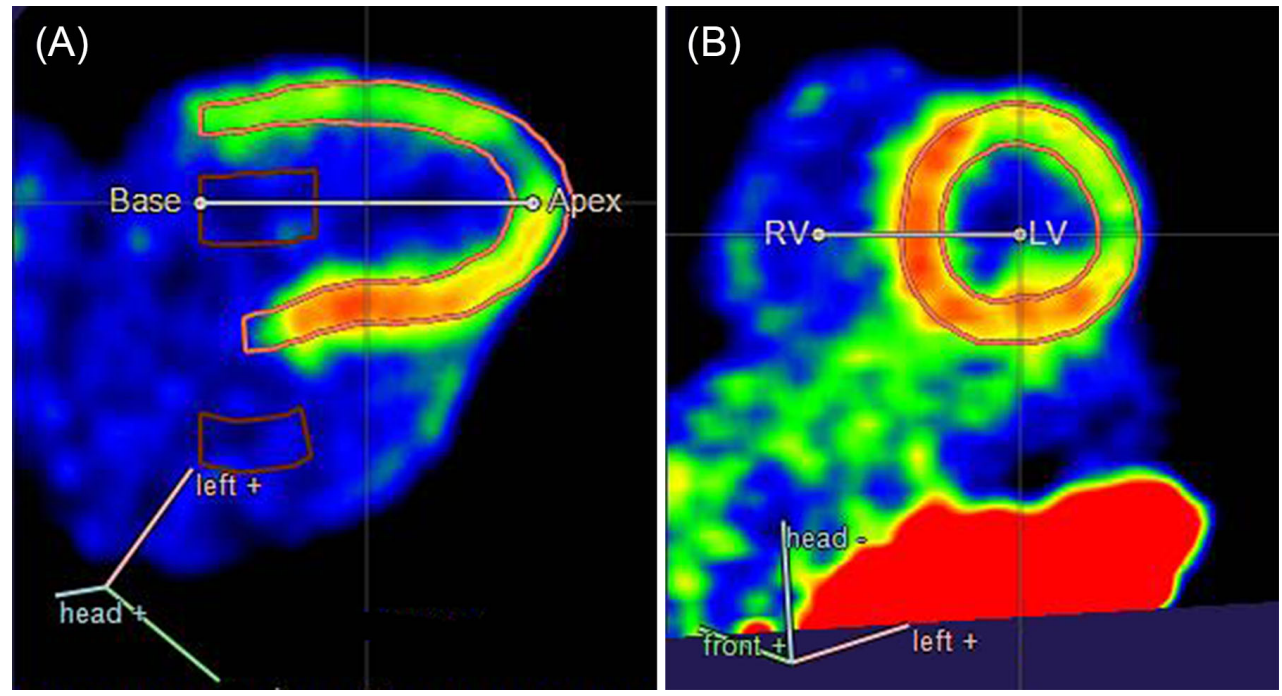

Fig. 1. Left ventricle myocardium segmentation on Carimas $^{T M}$ software, (A) Horizontal long axis, and (B) Short axis.

\subsection{Statistical analysis}

Continuous variables were evaluated for normal distribution using histograms and the Kolmogorov-Smirnov test and presented as mean and standard deviation and median and interquartile range. Categorical variables were summarized as frequency and percentage. The Chi-square test and Fisher's exact test were used to compare categorical variables between PD and non-PD patients. The independent samples $t$-test and the Mann-Whitney-Wilcoxon test were used to compare continuous variables between PD and non-PD patients. Multivariate logistic regression was used to

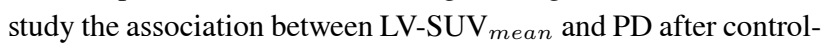
ling for potential confounders, including sex, age, hypertension, diabetes mellitus, ischemic heart disease, and uptake time. LV$\mathrm{SUV}_{\text {mean }}$ was entered into the regression in the first block, which was followed by an additional block in which all confounders were included. A backward method was applied for variable selection. The Wald test was used as criteria, and variables with a $P$-value $>0.1$ were removed. The area under the receiver operating characteristic curve (AUC) was applied to evaluate the ability of LV$\mathrm{SUV}_{\text {mean }}$ to discriminate between PD and non-PD patients. All statistical tests were 2 -sided, and a $P$-value $<0.05$ was considered statistically significant. SPSS was used for all statistical analyses (IBM Corp. Released in 2017. IBM SPSS Statistics for Windows, version 25.0. Armonk, NY: IBM Corp).

\section{Results}

Seventy-six patients were included in this study. Fifty-two patients were diagnosed with PD, and 24 subjects did not meet the UK Brain Bank criteria for PD (Hughes et al., 1992). Patients' characteristics are summarized in Table 1. The PD patients were younger than the non-PD patients $(P=0.014)$, with median ages of 64 and 69 years, respectively. The other evaluated characteristics were not significantly different between the two groups of patients. [18F] FDOPA PET scans of the brain identified characteristic reduced presynaptic dopaminergic activity in all PD patients as opposed to preserved dopaminergic activity in the nonPD group. The mean [18F] FDOPA LV-SUV mean $_{\text {was lower for }}$ the PD patients compared to the non-PD patients $(1.08 \pm 0.21$ vs. $1.24 \pm 0.32, P=0.015)$. In the multivariate regression, only IHD and LV-SUV mean remained in the last step of the regression, and a lower LV-SUV mean was independently associated with PD $\left(\mathrm{OR}_{a d j}=0.033,95 \%\right.$ CI 0.002-0.484, $\left.P=0.013\right)$. Overall, the LV$\mathrm{SUV}_{\text {mean }}$ was able to discriminate between PD and non-PD patients (AUC $=0.641, P=0.049$ ).

Examples of the brain and cardiac [18F] FDOPA PET scans of patients diagnosed with and without PD are depicted in Fig. 2.

\section{Discussion}

This proof-of-concept study demonstrates the feasibility of utilizing quantitative [18F] FDOPA PET scans of the heart for discriminating between patients with PD from patients without PD. To date, the gamma-emitting tracer [123I]-MIBG is the only tracer in current clinical practice for noninvasive demonstration of cardiac denervation (Dhawan et al., 2002; Sudmeyer et al., 2011; Uyama et al., 2017; Yoshii et al., 2017). However, [123I]-MIBG is a false analog of norepinephrine that differs from true catecholamines. Therefore it is not as efficiently removed by sympathetic nerves and does not undergo intracellular metabolism by monoamine oxidase or by catechol-O-methyltransferase (Balogova et al., 2013; Neurauter et al., 2008). The main limitation of [123I]-MIBG is that it is not a positron emitter, but is instead imaged employing a gamma camera which has inherent limitations, such as reduced spatial resolution, increased radiation dose, and longer acquisition, compared with PET imaging (Goldstein, 2013).

$6-[18 \mathrm{~F}]$-fluorodopamine, is an investigational positron emitter that has been effectively used for visualizing cardiac sympathetic denervation (Goldstein and Holmes, 1997). However, it is not widely available, and, unlike [18F] FDOPA, it does not pass the blood-brain-barrier. It, therefore, cannot be used for imaging striatal dopaminergic uptake. [18F] FDOPA, an analog of L-dopa, the immediate precursor of dopamine, is a PET tracer used in nuclear departments for the evaluation of striatal dopaminergic integrity as part of the diagnostic work-up for PD. The ability to use this tracer for both striatal and cardiac imaging is appealing since it enables a "one-stop-shop" imaging procedure for patients being evaluated for PD. 
Table 1. Patients Characteristics $(n=76)$.

\begin{tabular}{|c|c|c|}
\hline Diagnosis & $n(\%)$ & \\
\hline PD & $52(68.4)$ & \\
\hline Non-PD & $24(31.6)$ & \\
\hline \multicolumn{3}{|c|}{ Age, yr. (median, IQR; mean $\pm \mathrm{SD})^{*}$} \\
\hline All patients & $65(58.2-72)$ & $63.2 \pm 12.6$ \\
\hline PD & $64(57-70)$ & $61.8 \pm 11.6$ \\
\hline Non-PD & $69(65-77.5)$ & $67.4 \pm 14.2$ \\
\hline \multicolumn{3}{|c|}{ BMI (median, IQR; mean \pm SD) } \\
\hline All patients & $26.2(23.6-29.3)$ & $26.7 \pm 4.1$ \\
\hline PD & $25.8(23-29.3)$ & $26.2 \pm 4.3$ \\
\hline Non-PD & $27.6(25.5-30.7)$ & $27.8 \pm 3.3$ \\
\hline \multicolumn{3}{|l|}{ Sex } \\
\hline Female & $25(32.9 \%)$ & \\
\hline Male & $51(67.1 \%)$ & \\
\hline \multicolumn{3}{|c|}{ LV-SUV_mean $(\text { median, IQR; mean } \pm \mathrm{SD})^{*}$} \\
\hline All patients & $1.14(0.95-1.25)$ & $1.13 \pm 0.3$ \\
\hline PD & $1.12(0.9-1.2)$ & $1.08 \pm 0.2$ \\
\hline Non-PD & $1.19(1-1.5)$ & $1.24 \pm 0.3$ \\
\hline $\mathrm{DM}^{* *}$ & $13(17.8 \%)$ & \\
\hline PD & $9(18 \%)$ & \\
\hline Non-PD & $4(17.4 \%)$ & \\
\hline $\mathrm{IHD}^{* *}$ & $6(8.2 \%)$ & \\
\hline PD & $2(4 \%)$ & \\
\hline Non-PD & $4(17.4 \%)$ & \\
\hline
\end{tabular}

In the present trial, quantitative analysis of [18F] FDOPA uptake in the left ventricle myocardium had only a weak ability to differentiate between patients with PD and those without. One possible explanation is the heterogeneity of the comparison group. Another explanation for this could be the lack of clinical data available on symptoms related to autonomic failure in the study cohort. Previous studies have shown that about $30-40 \%$ of PD patients have OH (Merola et al., 2018; Velseboer et al., 2011) and that imaging cardiac sympathetic denervation is best for differentiating patients with PD and OH from patients with MSA (Goldstein et al., 2008). In contrast, cardiac sympathetic denervation in PD patients without $\mathrm{OH}$ is variable (Li et al., 2002). Thus, the current study cohort probably consisted of PD patients, both with and without $\mathrm{OH}$, showing varying degrees of cardiac denervation.

Nevertheless, these results show promise that cardiac [18F] FDOPA uptake may be able to aid in the workup of patients with suspected PD, and allow differentiation between those with idiopathic PD and non-neurodegenerative parkinsonism. Another limitation is that a group of patients with an established clinical diagnosis of MSA was not available as part of the study population. One would expect such patients to generally have normal values of cardiac [18F] FDOPA uptake, and that those values would be higher than those measured in PD patients. Such an analysis should be pursued in future studies to verify whether [18F] FDOPA can genuinely serve as a clinically relevant biomarker for cardiac sympathetic denervation that could differentiate between PD and MSA patients.
In conclusion, this proof-of-concept study demonstrates the feasibility of differentiating between patients with PD and without PD by quantifying cardiac [18F] FDOPA uptake. This finding should be verified in more extensive prospective trials.

\section{Abbreviations}

AUC: Area under the receiver operating characteristic curve; ET: Essential tremor; L-dopa: L-dihydroxyphenylalanine; LVSUV $V_{\text {mean }}$ : Left ventricular mean standardized uptake value; MSA: Multiple system atrophy; OH: Orthostatic hypotension; PD: Parkinson's disease; PET: Positron emission tomography; PET/CT: Positron emission tomography/computed-tomography; SPECT: Single photon emission computed tomography; [123I]MIBG: [123I]-metaiodobenzylguanidine; [18F] FDOPA: L-3,4dihydroxy-6-[18F] fluoro-phenylalanine.

\section{Author contributions}

JK contributed to the conception of the study, data collection, and analysis, study design, statistical analysis, writing and revising the manuscript; AL contributed to data collection and analysis and revising the manuscript; MK, TS, and NG contributed to the study design, data analysis, writing and revising the manuscript; $\mathrm{NF}$ contributed to data collection and analysis, study design, statistical analysis and revising the manuscript; HL and EES contributed to the conception of the study, data collection, study design, writing and revising the manuscript; All authors read and approved the final manuscript. 

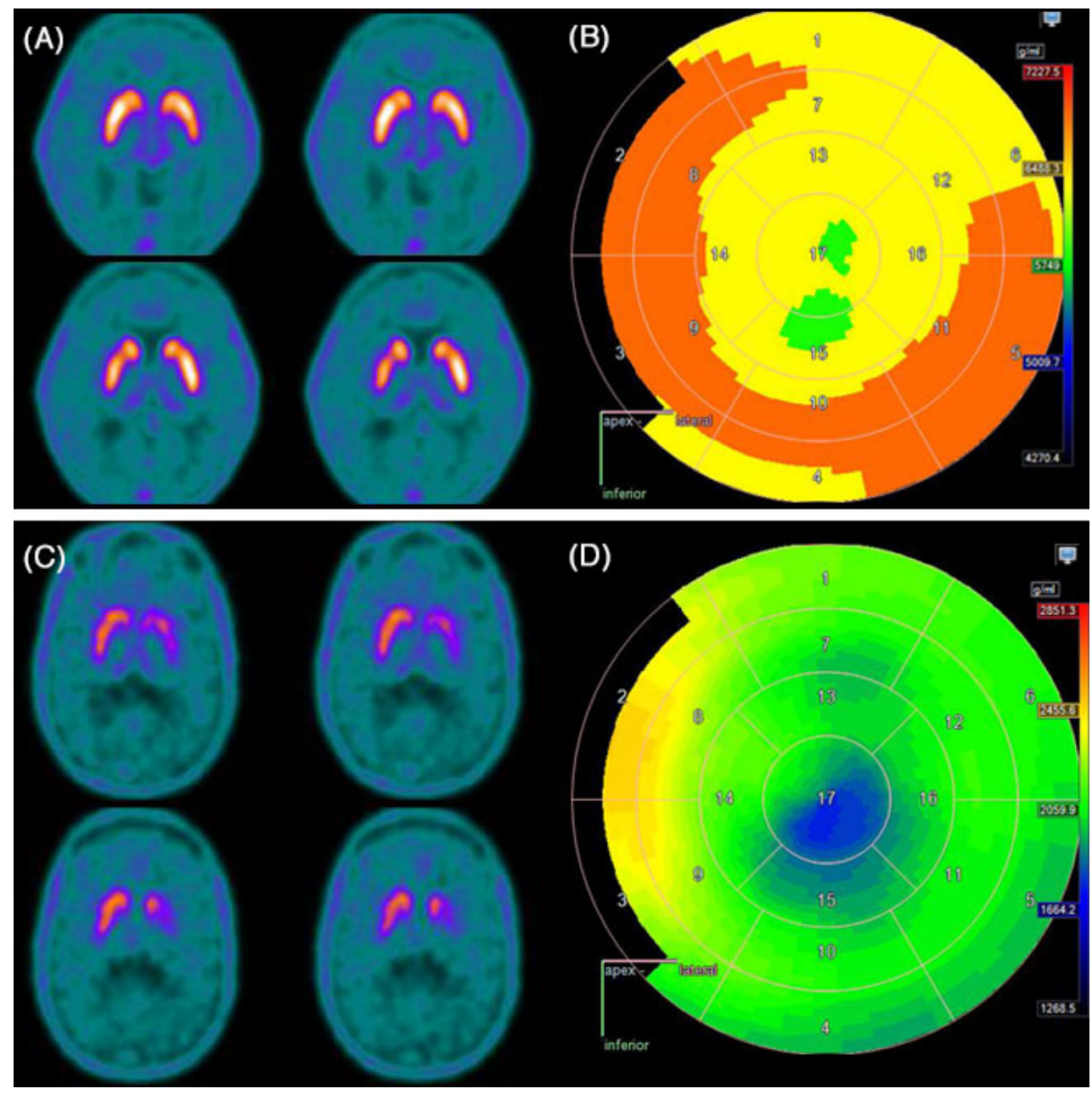

Fig. 2. [18F] FDOPA PET scans of two patients who presented with tremor and referred for differentiation between essential tremor and PD. (A \& C) Transaxial brain [18F] FDOPA PET. (B \& D) Polar view of cardiac [18F] FDOPA PET generated by Carimas ${ }^{T M}$ software. (A \& B) A 69-year-old patient with normal [18F] FDOPA uptake in the basal ganglia, compatible with essential tremor. The cardiac LV-SUV mean was 1.49. (C \& D) A 38year-old patient with markedly decreased [18F] FDOPA uptake in the left striatum compatible with the dominant right-sided clinical manifestations and consequently diagnosed as PD. The cardiac LV-SUV mean was 0.7 .

\section{Ethics approval and consent to participate}

All procedures performed in studies involving human participants were in accordance with the ethical standards of the institutional and/or national research committee and with the 1964 Helsinki declaration and its later amendments or comparable ethical standards.

This study was approved by the Tel-Aviv Sourasky Medical Center IRB (approval number 0606-14-TLV). All subjects signed informed consent.

\section{Acknowledgments}

We thank our dedicated technicians for their contribution to this work.

\section{Conflict of Interest}

The authors declare no conflict of interest.

Submitted: June 18, 2020

Received: July 14, 2020

Accepted: July 24, 2020

Published: September 30, 2020

\section{References}

Balogova, S., Talbot, J., Nataf, V., Michaud, L., Huchet, V., Kerrou, K. and Montravers, F. (2013) 18F-Fluorodihydroxyphenylalanine vs. other radiopharmaceuticals for imaging neuroendocrine tumours according to their type. European Journal of Nuclear Medicine and Molecular Imaging 40, 943-966.

Braak, H., Ghebremedhin, E., Rüb, U., Bratzke, H. and Del Tredici, K. (2004) Stages in the development of Parkinson's disease-related pathology. Cell and Tissue Research 318, 121-134.

Braune, S. (2001) The role of cardiac metaiodobenzylguanidine uptake in the differential diagnosis of parkinsonian syndromes. Clinical Autonomic Research 11, 351-355.

Dhawan, V., Ma, Y., Pillai, V., Spetsieris, P., Chaly, T. and Eidelberg, D. (2002) Comparative analysis of striatal FDOPA uptake in Parkinson's disease: ratio method versus graphical approach. Journal of Nuclear Medicine 43, 1324-1230.

Druschky, A., Hilz, M. J., Platsch, G., Radespiel-Tröger, M., Druschky, K., Kuwert, T. and Neundörfer, B. (2000) Differentiation of Parkinson's Disease and multiple system atrophy in early disease stages by means of I-123-MIBG-SPECT. Journal of the Neurological Sciences 175, 312. 
Eshuis, S. A., Maguire, R. P., Leenders, K. L., Jonkman, S. and Jager, P. L. (2006) Comparison of FP-CIT SPECT with F-DOPA PET in patients with de novo and advanced Parkinson's Disease. European Journal of Nuclear Medicine and Molecular Imaging 33, 200-209.

Garnett, E. S., Firnau, G. and Nahmias, C. (1983) Dopamine visualized in the basal ganglia of living man. Nature 305, 137-138.

Goldstein, D. S. (2013) Sympathetic neuroimaging. Handbook of Clinical Neurology 117, 365-370.

Goldstein, D. S. and Holmes, C. (1997) Metabolic fate of the sympathoneural imaging agent $6-[18 \mathrm{~F}]$ fluorodopamine in humans. Clinical and Experimental Hypertension 19, 155-161.

Goldstein, D. S., Holmes, C., Bentho, O., Sato, T., Moak, J., Sharabi, Y., Imrich, R., Conant, S. and Eldadah, B. A. (2008) Biomarkers to detect central dopamine deficiency and distinguish Parkinson disease from multiple system atrophy. Parkinsonism and Related Disorders 14, 600607.

Goldstein, D. S., Holmes, C., Frank, S. M., Dendi, R., Cannon, R. O., Sharabi, Y., Esler, M. D. and Eisenhofer, G. (2002) Cardiac sympathetic dysautonomia in chronic orthostatic intolerance syndromes. Circulation 106, 2358-2365.

Goldstein, D. S., Holmes, C., Lopez, G. J., Wu, T. and Sharabi, Y. (2018) Cardiac sympathetic denervation predicts PD in at-risk individuals. Parkinsonism and Related Disorders 52, 90-93.

Goldstein, D. S., Kopin, I. J., Sharabi, Y. and Holmes, C. (2015) Plasma biomarkers of decreased vesicular storage distinguish Parkinson disease with orthostatic hypotension from the parkinsonian form of multiple system atrophy. Clinical Autonomic Research 25, 61-67.

Goldstein, D. S., Sewell, L. and Holmes, C. (2010) Association of anosmia with autonomic failure in Parkinson Disease. Neurology 74, 245-251.

Goldstein, D. S., Sewell, L. and Sharabi, Y. (2011) Autonomic dysfunction in PD: a window to early detection? Journal of the Neurological Sciences 310, 118-122.

Goldstein, D. S., Sharabi, Y., Karp, B. I., Bentho, O., Saleem, A., Pacak, K. and Eisenhofer, G. (2007) Cardiac sympathetic denervation preceding motor signs in Parkinson disease. Clinical Autonomic Research 17, 118-121.

Goldstein, D. S., Sullivan, P., Holmes, C., Miller, G. W., Sharabi, Y. and Kopin, I. J. (2014) A vesicular sequestration to oxidative deamination shift in myocardial sympathetic nerves in Parkinson's Disease. Journal of Neurochemistry 131, 219-228.

Hughes, A. J., Daniel, S. E., Kilford, L. and Lees, A. J. (1992) Accuracy of clinical diagnosis of idiopathic Parkinson's Disease: a clinicopathological study of 100 cases. Journal of Neurology, Neurosurgery \& Psychiatry 55, 181-184.

Iwasa, K., Nakajima, K., Yoshikawa, H., Tada, A., Taki, J. and Takamori, M. (1998) Decreased myocardial123I-MIBG uptake in Parkinson's Disease. Acta Neurologica Scandinavica 97, 303-306.

Jacobson, A. F., Senior, R., Cerqueira, M. D., Wong, N. D., Thomas, G. S., Lopez, V. A., Agostini, D., Weiland, F., Chandna, H. and Narula, J. (2010) Myocardial Iodine-123 Meta-Iodobenzylguanidine imaging and cardiac events in heart failure. Journal of the American College of Cardiology 55, 2212-2221.

Kim, J. S., Park, H. E., Park, I. S., Oh, Y. S., Ryu, D. W., Song, I. U., Jung, Y. A., Yoo, I. R., Choi, H. S., Lee, P. H. and Lee, K. S. (2017) Normal 'heart' in Parkinson's Disease: is this a distinct clinical phenotype? European Journal of Neurology 24, 349-356.

Koller, W. C. (1992) When does Parkinson's Disease begin? Neurology 42, 27-28.

Li, S., Dendi, R., Holmes, C. and Goldstein, D. S. (2002) Progressive loss of cardiac sympathetic innervation in Parkinson's Disease. Annals of Neurology 52, 220-223.

Lovenberg, W., Weissbach, H. and Udenfriend, S. (1962) Aromatic Lamino acid decarboxylase. The Journal of Biological Chemistry 237, 89-93.

Manabe, Y., Inui, Y., Toyama, H. and Kosaka, K. (2017) 123Imetaiodobenzylguanidine myocardial scintigraphy with early images alone is useful for the differential diagnosis of dementia with Lewy bodies. Psychiatry Research: Neuroimaging 261, 75-79.

Merola, A., Sawyer, R. P., Artusi, C. A., Suri, R., Berndt, Z., LopezCastellanos, J. R., Vaughan, J., Vizcarra, J. A., Romagnolo, A. and Espay, A. J. (2018) Orthostatic hypotension in Parkinson Disease: impact on health care utilization. Parkinsonism and Related Disorders 47, 45-49.

Miyamoto, T., Miyamoto, M., Inoue, Y., Usui, Y., Suzuki, K. and Hirata, K. (2006) Reduced cardiac 123I-MIBG scintigraphy in idiopathic REM sleep behavior disorder. Neurology 67, 2236-2238.

Nanni, C., Fanti, S. and Rubello, D. (2007) 18F-DOPA PET and PET/CT. Journal of Nuclear Medicine 48, 1577-1579.

Nesterov, S. V., Han, C., Mäki, M., Kajander, S., Naum, A. G., Helenius, H., Lisinen, I., Ukkonen, H., Pietilä, M., Joutsiniemi, E. and Knuuti, J. (2009) Myocardial perfusion quantitation with 15O-labelled water PET: high reproducibility of the new cardiac analysis software (Carimas $^{\mathrm{TM}}$ ). European Journal of Nuclear Medicine and Molecular Imaging 36, 1594-1602.

Neurauter, G., Schrocksnadel, K., Scholl-Burgi, S., Sperner-Unterweger, B., Schubert, C., Ledochowski, M. and Fuchs, D. (2008) Chronic immune stimulation correlates with reduced phenylalanine turnover. $\mathrm{Cur}$ rent Drug Metabolism 9, 622-627.

Oka, H., Mochio, S., Onouchi, K., Morita, M., Yoshioka, M. and Inoue, K. (2006) Cardiovascular dysautonomia in de novo Parkinson's Disease. Journal of the Neurological Sciences 241, 59-65.

Oka, H., Toyoda, C., Yogo, M. and Mochio, S. (2011) Cardiovascular dysautonomia in de novo Parkinson's Disease without orthostatic hypotension. European Journal of Neurology 18, 286-292.

Orimo, S., Amino, T., Itoh, Y., Takahashi, A., Kojo, T., Uchihara, T., Tsuchiya, K., Mori, F., Wakabayashi, K. and Takahashi, H. (2005) Cardiac sympathetic denervation precedes neuronal loss in the sympathetic ganglia in Lewy body disease. Acta Neuropathologica 109, 583-588.

Orimo, S. (2002) Sympathetic cardiac denervation in Parkinson's Disease and pure autonomic failure but not in multiple system atrophy. Journal of Neurology, Neurosurgery and Psychiatry 73, 776-776.

Orimo, S., Ozawa, E., Oka, T., Nakade, S., Tsuchiya, K., Yoshimoto, M., Wakabayashi, K. and Takahashi, H. (2001) Different histopathology accounting for a decrease in myocardial MIBG uptake in PD and MSA. Neurology 57, 1140-1141.

Orimo, S., Uchihara, T., Nakamura, A., Mori, F., Kakita, A., Wakabayashi, K. and Takahashi, H. (2008) Axonal alpha-synuclein aggregates herald centripetal degeneration of cardiac sympathetic nerve in Parkinson's Disease. Brain: A Journal of Neurology 131, 642-650.

Raffel, D. M., Koeppe, R. A., Little, R., Wang, C., Liu, S., Junck, L., Heumann, M. and Gilman, S. (2006) PET measurement of cardiac and nigrostriatal denervation in Parkinsonian syndromes. Journal of $\mathrm{Nu}$ clear Medicine 47, 1769-1777.

Sudmeyer, M., Antke, C., Zizek, T., Beu, M., Nikolaus, S., Wojtecki, L., Schnitzler, A. and Muller, H. (2011) Diagnostic accuracy of combined FP-CIT, IBZM, and MIBG scintigraphy in the differential diagnosis of degenerative Parkinsonism: a multidimensional statistical approach. Journal of Nuclear Medicine 52, 733-740.

Uyama, N., Otsuka, H., Shinya, T., Otomi, Y., Harada, M., Sako, W., Izumi, Y., Kaji, R., Watanabe, Y., Takashi, S. and Kunikane, Y. (2017) The utility of the combination of a SPECT study with [123I]-FP-CIT of dopamine transporters and [123I]-MIBG myocardial scintigraphy in differentiating Parkinson disease from other degenerative parkinsonian syndromes. Nuclear Medicine Communications 38, 487-492.

Velseboer, D. C., de Haan, R. J., Wieling, W., Goldstein, D. S. and de Bie, R. M. A. (2011) Prevalence of orthostatic hypotension in Parkinson's disease: a systematic review and meta-analysis. Parkinsonism \& Related Disorders 17, 724-729.

Yoshii, F., Ryo, M., Baba, Y., Koide, T. and Hashimoto, J. (2017) Combined use of dopamine transporter imaging (DAT-SPECT) and 123 Imetaiodobenzylguanidine (MIBG) myocardial scintigraphy for diagnosing Parkinson's Disease. Journal of the Neurological Sciences 375, $80-85$ 